\title{
Allergy as precursor of carcinogenesis
}

\author{
Robert Skopec* \\ Department 01, AXON, Slovakia
}

\begin{abstract}
The fact that cholinergic neurons inhibit acute inflammation qualitatively expanded the mechanism of how the nervous system modulates immune responses. The nervous system reflexively controls and regulates the inflammatory response in real time. It means that we could use above knowledge for the treatment of the inflammation at the level of the hard-wired neural systems. The inflammatory response is crucial, because the insufficient response cause immunodeficiency, what can lead to infection and cancer. Excessive response can cause morbidity and mortality through lymphoid organs (spleen, gut), where conversion of allergy into carcinogenesis is ongoing. Our point is that study of the tumor microenvironment is no longer enough for mechanistic explanation of carcinogenesis. As a prior mechanism newly must be seen lymphoid organs and regulation of the immune system by the CNS.
\end{abstract}

\section{Introduction}

The latest surveys show that the rates of allergy are increasing throughout the world, affecting up to $30-35 \%$ of people at some stage in their lives. This increase was initially seen in countries such as the Europe and USA, but can now be found in all countries undergoing industrial development. The pattern of allergy is also changing initially, the increase was in asthma and allergic rhinitis (hay fever). However, recent studies have confirmed a significant increase in the incidence of food allergies, in particular amongst children.

Allergy is caused when the body's immune system reacts to a normally harmless substance, such as pollen, food, or house dust mite. The body identifies the substance as a threat and produces an inappropriate, exaggerated response to it. What we are only beginning to understand is what tips the balance in favour of allergy. Researchers have suggested that a number of factors might cause someone to become allergic:

Genetics: within the human population, changes in genes take many hundreds of years to cause consequences in disease. So atopy/ genetic tendency alone cannot account for the current increase in allergy seen over the past few decades. Current research is investigating the effect of the environment in which we live on genetic factors. It is now clear that many genes can be 'turned on or off' by environmental factors. For example, viral infections are able to modulate our immune systems by switching certain genes on or off, promoting an allergic tendency in immune cells.

The Hygiene Hypothesis: this suggests that the immune system needs to come into contact with a variety of micro-organisms and bacteria while it is developing at the infant stage, in order that it responds appropriately later in life. We now live in an environment where we use cleaners containing anti-microbial agents, and food preparation is more hygienic than ever. Changes in the foods we eat: a number of research teams are investigating whether reduced levels of nutrients - in particular vitamin D, omega-3 fatty acids (in fish) or antioxidants - might contribute to the development of allergy. While a diet low in oily fish has been associated with increased risk of childhood asthma and allergies in some studies, one study giving extra fish oils to babies did not prevent the occurrence of allergies [1].
Environmental factors: our environment today is very different from 50 years ago. While there is evidence that pollutants can exacerbate existing airway allergy, the question of whether pollution can cause new allergy remains controversial. One hypothesis for which there are accumulating data, is that the increase in allergy mirrors our declining exposure to bacteria and other micro-organisms in our environment.

Allergies are classified into IgE mediated and non-IgE mediated allergies. In IgE mediated allergies the immune system produces exaggerated amounts of a distinct class of antibodies known as $\operatorname{IgE}$ antibodies that are, specific for the particular offending allergens [2]. These IgE antibodies bind to the surface of cells in the body called mast cells which become 'IgE-sensitised' such that these cells can then identify particular allergens the next time they come in contact with the body. This process is called sensitisation, and at this stage there are no physical symptoms of an allergy. Mast cells are present in tissues that are in contact with the external environment, including the skin, nose, eyes, mouth, throat, stomach and gut. The next time that the same allergen is encountered the mast cells identify it as an intruder and produce histamine and other chemicals.

\section{How the nervous system integrates an inflammatory response}

The autonomic nervous system interacts with the brain including the limbic system, brain stem and hypothalamus. Hypothalamic neural output is connected to sympathetic and parasympathetic nuclei in the brain stem and spinal cord. Hormonal output controls the release pituitary hormones, they in turn regulate basic functions of the endocrine organs. Autonomic nervous functions are subconscious, and can be placed under conscious control by signals originating in

Correspondence to: Robert Skopec, Research Analyst, Department 01, AXON, Slovakia, Tel: +421 908220692; E-mail: zxcbnvm7@gmail.com

Key words: allergy, inflammation, TNF, bile acids, antioxidants, Sanorin, carcinogenesis, antihistamines

Received: January 08, 2016; Accepted: January 26, 2016; Published: January 29, 2016 
the higher brain (cerebral cortex). Basic neural pathway reflexively monitors and adjusts the inflammatory response relay information to the hypothalamus. The nervous system integrates the inflammatory response: gathers information about invasive events, mobilizes defences, creates memory to increase chances for survival. The neural control of acute inflammation is reflexive, directly interconnected and controllable. The cholinergic anti-inflammatory mechanisms inhibit the activation of macrophages and the release of cytokines [3].

Evidence is indicating that the stimulation of the vagus nerve by electrical or pharmacological means prevents inflammation and inhibits the release of cytokines [4]. Tumor-necrosis factor (TNF) as a cytokine amplifies and prolongs the inflammatory response by activating other cells to release: cytokines such as interleukin 1 (IL-1) and high mobility group B1 (HMGB1), mediators like eicosanoids, nitric oxide and reactive oxygen species during invasion and cancer [4].

Neural inhibition of inflammation primarily means the cholinergic inhibition of TNF. These neural anti-inflammatory mechanisms also inhibit the release of IL-1, IL-18 and HMGB1. Anti-inflammatory cytokines, such as IL-10 and transforming growth factor- $\beta$ (TGF- $\beta$ ) specifically inhibit the release of TNF and other proinflammatory mediators. The classical stress hormones (adrenal glucocorticoids, adrenaline, etc.) inhibit cytokine synthesis and intracellular signal transduction [3]. These findings show that a loss of endogenous anti-inflammatory mechanisms converts the protective, self-limited inflammatory response into excessive, potentially deleterious response (cancer).

There are also evidences that inflammatory stimuli can activate anti-inflammatory signals from the central nervous system (CNS). The inflammation in peripheral tissues alters neuronal signaling in hypothalamus [5]. This communication is bi-directional, because cytokines can activate hypothalamic-pituitary release of glucocorticoids and, in turn, glucocorticoids suppress further cytokines synthesis. The cells of the immune system can produce neuropeptides (including endorphins), acetylcholines and other neurotrasnsmitters. These facts show the importance of the interaction between the nervous system and immune system signaling [5].

\section{The cholinergic anti-inflammatory pathway}

The DNA damage was evaluated by "the comet assay"or single cell gel electrophoresis (SCGE), both in its conventional use and by the Endonuclease III method, which allow to detect the presence of oxidized pyrimidines [6].

Bile acids (CDA and CDCA) resulted genotoxic on both normal and tumor human cells. The inclusion of the endonuclease III digestion step in the comet assay demonstrated that bile acids induced an oxidative DNA damage. On the other side, treatment of colonocytes with bile acids in the presence of the antioxidants caused a reduction of DNA damage [7].

The neural mechanisms inhibiting macrophage activation through parasympathetic outflow is the cholinergic anti-inflammatory pathway. Acetylcholine (parasympathetic neurotransmitter) effectively deactivates macrophages $[5,8]$.

The vagus nerve innervates the organs of the reticuloendothelial system (liver, lung, spleen, kidneys and gut). In experimental activation of cholinergic anti-inflammatory pathway by direct electrical stimulation the efferent vagus nerve inhibits the synthesis of TNF in liver, spleen, heart and attenuates serum concentrations of TNF in endotoxaemia. This represents a hard-wired connection between nervous and immune systems function.

Direct stimulation of the vagus nerve in situ inhibits proinflammatory cytokines in liver and cardiac tissue. Stimulation of the right, or left cervical vagus nerves protects against the development of hypotension and inhibits serum TNF responses. This protection is dependent on the applied voltage.

The molecular dovetail between the cholinergic nervous system and innate immune system is a nicotinic, $\alpha$-bungarotoxin-sensitive macrophage acetylcholine receptor.

Tissue macrophages produce most of the TNF during inflammatory response. Exposure of human macrophages to nicotine or acetylcholine inhibits release of TNF, IL-1, and IL-18 in response to endotoxine. Vagus nerve stimulation inhibits the inflammatory response. Activation of the cholinergic receptor transduces intracellular signals that inhibit cytokines synthesis at a post-transcriptional stage. Other non-neuronal cells producing acetylcholine (epithelial cells, T-lymphocytes, and endothelial cells) also participate in modulating the function of adjacent tissue macrophages [1].

Neural regulation of discrete, distributed, localized inflammatory sites provides a mechanism for integrating responses in real time. Electrical stimulation of the vagus nerve in humans is showing that memory formation and vagus nerve activity are closely linked [5].

The CNS receives sensory inputs from the immune system by humoral and neural pathways. The immune system functions like a sixth sense which detects microbial invasion and produces molecules that transmitting this information to the brain. TNF and other immune mediators use access to brain centers devoid of a blood-brain barrier in the circumventricular region. The dorsal vagal complex including the sensory nuclei of the solitary tract, the area postrema and the dorsal motor nucleus of the vagus responds to increased circulating amounts of TNF by altering motor activity in the vagus nerve [2,9].

These neural inflammation-sensing pathways function at low thresholds of detection and can activate responses also when the inflammatory agents are present in tissue in small quantities which are not high enough to reach the brain by bloodstream. The afferent vagus pathway is activated by very low doses of endotoxin or IL-1, while higher doses of these agents can directly activate thermogenic responses through the humoral pathway to the brain. Neurons in the vagus nerve express IL-1 receptor mRNA and dicrete IL-1-binding sites have been identified on glomus cells in the vagus nerve proper. The first CNS synapse for afferent vagus signals are in the nucleus tractus solitaries, and electrolytic lesioning of this region impairs the development of IL-1-induced fever. The inflammation-derived sensory input can be processed differentially in the brain $[2,5,10]$.

\section{Systemic anti-inflammatory response to allergy and cancer}

The vagus nerve activity relay to the medullary reticular formation, to the locus coruleus and to the hypothalamus, and lead to increased release of ACTH from the anterior pituitary. Here is in the tumor microenvironment also the overlap between allergy and cancer through expansion of the myeloid-derived suppressor cells (MDSC) into the medullary and extramedullary reticular formation. The inflammatory reflex as a localized, rapid and discrete mechanism can also induce a systemic humoral anti-inflammatory response to allergy and cancer [11]. 
The vagus nerve innervates the organs of the reticuloendothelial system (liver, lung, spleen, kidneys and gut).

In experimental activation of cholinergic anti-inflammatory pathway by direct electrical, or pharmacological stimulation the efferent vagus nerve inhibits the synthesis of TNF in liver, spleen, heart and attenuates serum concentrations of TNF in endotoxaemia [1].

Increased cytokine production in tissues causes pain, showing another mechanism for transferring information from the immune system to the brain. This information is relayed to the other brain centers influencing motor output in the vagus nerve. Pain and stress activate the flight-or-fight responses, leading to increase of adrenaline and noradrenaline, what can inhibit macrophage activation and suppress synthesis of TNF and other cytokines. High sympathetic activity caused increase in catecholamines stimulate the $\beta$-adrenergicreceptor-dependent release of IL-10, a potent anti-inflammatory cytokine from monocytes. The combined actions of these neural systems is anti-inflammatory by preventing spillover of lethal toxins into the circulation in both local (neural) and systemic (humoral) antiinflammatory mechanisms $[2,5,10]$.

Sporadic colon cancer is caused predominantly by dietary factors: first of all by meat consumption produced fats and mast cells. During our case studies we have selected bile acids as a risk factors correlated with fats and mast cells caused allergy $[2,5,10]$.

This was correlated with high levels of hydrophobic bile acids known from the Western-style diet and play plays a key role in colon carcinogenesis [11].

We can confirm that bile-acid-induced stresses cause cell death in susceptible cells contribute to genomic instability in surviving cells, impose Darwinian selection on survivors and enhance initiation and progression of colon cancer. The most likely major mechanisms by which hydrophobic bile acids induce stresses on cells are: the DNA damage, endoplasmic reticulum stress, and mitochondrial damage $[6,7]$.

Persistent exposure of colon epithelial cells to hydrophobic bile acids can result in the activation of pro-survival stress-response pathways, and the modulation of numerous genes/proteins associated with chromosome maintenance and mitosis. The multiple mechanisms by which hydrophobic bile acids contribute to genomic instability include: oxidative DNA damage, p53 and other mutations, micronuclei formation and aneuploidy [6,7].

A new research report suggests that antihistamines have significant anti-cancer properties because they interfere with the myeloid-derived suppressor cells (MDSC) which is known to reduce organism's ability to fight tumors. MDSCs are immune cells that inhibit T cells proliferation and activation. In healthy subjects immature myeloid cells develop in the bone marrow, and differentiate to develop into dendritic cells, macrophages and neutrophils [11].

In the presence of chronic inflammatory conditions (viral and bacterial infections) or cancer, myeloid differentiation leads to an expansion of MDSCs. They infiltrate inflammation sites and tumors through stopping the immune responses by inhibiting $\mathrm{T}$ cells and Natural Killer (NK) cells. MDSCs also support cancer cell proliferation, progression, metastasis and survival by promoting evasion from immune attack. Clinical research has found that cancer tissues containing high numbers of MDSCs become resistant to anti-cancer therapies and are associated with poor prognosis [11].

\section{One promising allergy and cancer therapy from Slovakia}

Above research draws a connection between commonly linked two diseases: allergy and cancer. Despite the link is relatively novel, the antihistamines can be used effectively in cancer therapies. Treatment with anti-histamines reversed the effects of myeloid derived suppressor cells. The research examined blood from patients with allergy symptoms (with increased histamine release) had higher levels of circulating MDSCs compared to non-allergic subjects.

Information about the inflammatory reflex and the cholinergic anti-inflammatory pathway is giving insight into physiological pathways and therapeutic strategies. I have managed research of the inflammatory reflex firstly on myself personally.

To activate neural anti-inflammatory mechanisms we have used a drug named Sanorin (Naphasolinium Nitrate 0,01 g) which is produced by Teva Czech Industries s.r.o., Opava, Czech Republic. It is available also in my home The Slovak Republic. Sanorin have initiated signals in proximal components of the pathway in the CNS, while acted as of macrophage activation, TNF synthesis + release, and inhibition of inflammatory responses in local and systemic inflammation during symptoms of the nasal allergy.

Recent evidence has shown that the TNF-suppressing activities of Sanorin in vivo are dependent on the cholinergic anti-inflammatory pathway, and that Sanorin functions as a pharmacological stimulator of the vagus nerve [5].

The mechanism through which Sanorin activates the vagus nerve is still under research: an increased vagus nerve firing has been observed after intracerebral administration. An effect may be dependent on specific CNS receptors. Sanorin also functions as a cathetrisator of the blood and lymph pathways.

Similarly specific anti-inflammatory responses have been observed also in response to intracerebral application of salicylates [12]. Systemic administration of the non-steroidal anti-inflammatory drugs aspirin (in Slovakia: Acylpirin), indomethacin and ibuprofen substantially increases vagus nerve activity [5].

The cholinergic anti-inflammatory pathways raise the probability that the vagus-nerve-stimulating activity of Sanorin and other above agents contribute to their anti-inflammatory action. Further research of the CNS receptors, pathways and neural mechanisms that activate the vagus nerve to inhibit production of TNF will facilitate development of this pharmacological vagus-nerve-stimulating approach in the therapy of allergy and cancer. Dyslipidemia and insulin resistance are commonly associated with catabolic or lipodystrophic conditions such as cancer and sepsis. Two common features of these metabolic disorders are adipose tissue dysfunction and elevated levels of tumour necrosis factor-alpha (TNF- $\alpha$ ). There is possible the multiple actions of this pro-inflammatory adipokine on adipose tissue biology. TNF- $\alpha$ can also impact the endocrine functions of adipose tissue. Taken together, TNF-a contributes to metabolic dysregulation by impairing both adipose tissue function and its ability to store excess fuel [13].

Dendritic cells (DC) are professional antigen-presenting cells uniquely suited for cancer immunotherapy. They induce primary immune responses, potentiate the effector functions of previously primed T-lymphocytes, and orchestrate communication between innate and adaptive immunity. The remarkable diversity of cytokine activation regimens, DC maturation states, and antigen-loading strategies 
employed in current DC-based vaccine design reflect an evolving, but incomplete, understanding of optimal DC immunobiology.

In the clinical realm, existing DC-based cancer immunotherapy efforts have yielded encouraging but inconsistent results. Despite recent U.S. Federal and Drug Administration (FDA) approval of DCbased sipuleucel-T for metastatic castration-resistant prostate cancer, clinically effective DC immunotherapy as monotherapy for a majority of tumors remains a distant goal. New identified strategies may allow for more potent "next-generation" DC vaccines. Additionally, multimodality approaches incorporating DC-based immunotherapy may improve clinical outcomes [14].

\section{An executive summary}

Since bile acids and oxidative stress decrease DNA repair proteins, this mechanism cause an increase in genomic instability. Our review is confirming a mechanistic explanation for important link between a Western-style diet, eat consumption, allergy and associated increased levels of (colon and other) cancers [6].

It is allowing to us to prophylactically administer antihistamines for cancer prevention. MSDCs and their products become as immunotherapy targets in cancer on the basis of seemingly related immune mechanism between allergy and carcinogenesis.

The chronic inflammation and allergy appears to be a precursor to growth of the cancer cells.

The vagus nerve activity relay to the medullary reticular formation, to the locus coruleus and to the hypothalamus, and lead to increased release of ACTH from the anterior pituitary.

Here is in the tumor microenvironment also the overlap between allergy and cancer through expansion of the myeloid-derived suppressor cells (MDSC) into the medullary and extramedullary reticular formation.

On the above basis we can hypothesize that the allergy is a first stage of the five cancer stages. The inflammatory reflex as a localized, rapid and discrete mechanism can also induce a systemic humoral antiinflammatory response to allergy and cancer.

The vagus nerve innervates the organs of the reticuloendothelial system (liver, lung, spleen, kidneys and gut). This a part of a hard-wired connection between nervous and immune systems function. The spleen is playing the role of a convertor of the allergy into the carcinogenesis by the mechanism of the inflammation.

These findings could result in a cell-based immunotherapy/vaccine development. For example, DC205+ activity has been considered as the starting point for inducing immune tolerance in inflammation resulting from abnormal $\mathrm{T}$-cell activity.

In experimental activation of cholinergic anti-inflammatory pathway by direct electrical, or pharmacological stimulation the efferent vagus nerve inhibits the synthesis of TNF in liver, spleen, heart and attenuates serum concentrations of TNF in endotoxaemia.

Our results suggests that meat eating leads to bile acids involvement in the tumor initiation by inducing a DNA oxidative damage and so add further evidences to the preventive properties of antioxidants (beta-carotene, and alpha-tocopherol, and Na-butyrate) [7].

Frequency and total consumption of red meat is a risk factor of colorectal cancer. Bile acids reduce the apoptosis-inducing effects of sodium butyrate on human colon adenoma (AA/C1) cells and this have an implications for colon carcinogenesis.

Our point is that study of the tumor microenvironment is no longer enough for mechanistic explanation of carcinogenesis. As a prior mechanism newly must be studied lymphoid organs and regulation of the immune system by the CNS.

One of the characteristics of the central nervous system was labeled the lack of a classical lymphatic drainage system. Although it is now accepted that the central nervous system undergoes constant immune surveillance that takes place within the meningeal compartment, the mechanisms governing the entrance and exit of immune cells from the central nervous system remain poorly understood.

In searching for $\mathrm{T}$-cell gateways into and out of the meninges, it was discovered functional lymphatic vessels lining the dural sinuses $[15,16]$. These structures express all of the molecular hallmarks of lymphatic endothelial cells, are able to carry both fluid and immune cells from the cerebrospinal fluid, and are connected to the deep cervical lymph nodes. The unique location of these vessels may have impeded their discovery to date, thereby contributing to the long-held concept of the absence of lymphatic vasculature in the central nervous system.

The discovery of the central nervous system lymphatic system may call for a reassessment of basic assumptions in neuroimmunology and sheds new light on the aetiology of neuroinflammatory and neurodegenerative diseases associated with immune system dysfunction. Evidence is showing that the therapeutic strategies must include not only the regulation of tumor microenvironment, but also the response potential of at the level of CNS.

So an intriguing possibility is that many of the above dietary and environmental factors may increase allergy risk by regulating genes which promote an allergic-type immune system. Hopefully, our understanding of epigenetics will increase over the coming years, offering new potential strategies by which we might be able to prevent allergy.

\section{References}

1. Borovikova LV, Ivanova S, Zhang M, Yang H, Botchkina GI, et al. (2000) Vagus nerve stimulation attenuates the systemic inflammatory response to endotoxin. Nature 405: 458-462. [Crossref]

2. Goehler LE, Gaykema RP, Hansen MK, Anderson K, Maier SF, et al. (2000) Vaga immune-to-brain communication: a visceral chemosensory pathway. Auton Neurosci 85: 49-59. [Crossref]

3. Ader R, Cohen N (2001) in Psychoneuroimmunology (eds. Ader R, Felten DL \& Cohen N) Academic, San Diego, 3-34.

4. Ben-Menachem E (2001) Vagus nerve stimulation, side effects, and long-term safety. J Clin Neurophysiol 18: 415-418. [Crossref]

5. Tracey KJ (2002) The inflammatory reflex. Nature 420: 853-859. [Crossref]

6. Payne CM, Bernstein C, Dvorak K, Bernstein H (2008) Hydrophobic bile acids, genomic instability, Darwinian selection, and colon carcinogenesis. Clin Exp Gastroenterol 1: 19-47. [Crossref]

7. Rosignoli P, Fabiani R, De Bartolomeo A, Fuccelli R, Pelli MA, et al. (2008) Genotoxic effect of bile acids on human normal and tumour colon cells and protection by dietary antioxidants and butyrate. Eur J Nutr 47: 301-309. [Crossref]

8. Tracey KJ, Czura CJ, Ivanova S (2001) Mind over immunity. FASEB J 15: 1575-1576 [Crossref]

9. Kawashima K, Fujii T (2000) Extraneuronal cholinergic system in lymphocytes. Pharmacol Ther 86: 29-48. [Crossref]

10. Blalock JE (1984) The immune system as a sensory organ. J Immunol 132: 1067-1070 [Crossref] 
11. Martin RK, Saleem SJ, Conrad DH et al., (2014) Mast cell histamine promotes the immunoregulatory activity of myeloid-derived suppressor cells. J Leuc Biol 96: 151.

12. Catania A, Arnold J, Macaluso A, Hiltz ME, Lipton JM (1991) Inhibition of acute inflammation in the periphery by central action of salicylates. Proc Natl Acad Sci U S A 88: 8544-8547. [Crossref]

13. Cawthorn WP, Sethi JK (2008) TNF-alpha and adipocyte biology. FEBS Lett 582: 117-131. [Crossref]
14. Datta J, Terhune JH, Lowenfeld L, Cintolo JA, Xu S, et al. (2014) Optimizing dendritic cell-based approaches for cancer immunotherapy. Yale J Biol Med 87: 491-518. [Crossref]

15. Louveau A, Smirnov I, Keyes TJ, Eccles JD, Rouhani SJ, et al. (2015) Structural and functional features of central nervous system lymphatic vessels. Nature 523: 337-341. [Crossref]

16. Mezey É, Palkovits M (2015) Neuroanatomy: Forgotten findings of brain lymphatics. Nature 524: 415. [Crossref]

Copyright: (C2016 Skopec R. This is an open-access article distributed under the terms of the Creative Commons Attribution License, which permits unrestricted use, distribution, and reproduction in any medium, provided the original author and source are credited. 